\title{
Antagonisme in vitro de Trichoderma spp. vis-à- vis de Rhizoctonia solani Kühn
}

\author{
Pierre CAMPOROTA \\ I.N.R.A., Station de Recherches sur la Flore pathogène dans le Sol, 17, rue Sully, F 21034 Dijon Cedex
}

RÉSUMÉ

\begin{abstract}
Cet article présente les résultats obtenus lors de la réalisation de la première étape d'un programme de sélection de souches de Trichoderma spp. utilisables pour la lutte biologique contre Rhizoctonia solani dans le sol : 28 souches de Trichoderma ont été confrontées in vitro à 3 souches de $R$. solani appartenant à des groupes d'anastomose différents. On a mesuré, pour chaque souche de Trichoderma, la capacité à envahir les colonies de l'agent pathogène ainsi que les 3 modes d'action : mycoparasitisme, émission de substances inhibitrices non volatiles et volatiles. Les 3 souches de $R$. solani ont montré des différences de sensibilité à l'antagoniste. L'effet inhibiteur de Trichoderma varie aussi en fonction des souches. Des observations au cours de l'expérimentation ainsi que des corrélations faites entre la capacité de colonisation de Trichoderma et les mesures des 3 modes d'action ont révélé que les souches de $R$. solani le plus rapidement détruites étaient celles touchées par le mycoparasitisme. L'étude de l'antagonisme in vivo en utilisant les mêmes souches pathogènes et antagonistes sera la deuxième étape de ce programme.
\end{abstract}

Mots clés additionnels : Antagonisme, mycoparasitisme, compétition, substances volatiles, substances non volatiles.

We present here results obtained in the first stage of a plan to select strains of Trichoderma spp. effective for biological control of Rhizoctonia solani in soils. 28 strains of Trichoderma species were tested in vitro for antagonism against 3 strains of $R$. solani belonging to 3 different anastomosis groups. The ability of each strain of Trichoderma to invade the colonies of the 3 different $R$. solani was measured on malt agar. Specific techniques were developed to measure the relative importance of the 3 main mechanisms of antagonism : mycoparasitism, and release of volatile or non-volatile compounds. The 3 strains of $R$. solani differed in their susceptibility to antagonism by Trichoderma. The 28 strains of Trichoderma differed in their antagonistic properties. Correlation between a Trichoderma's capacity to invade the colonies of $R$. solani and the measures of each mechanism of antagonism showed that mycoparasitism was the most effective mechanism for destroying the hyphae of $R$. solani. The same 28 strains of Trichoderma, well characterized in vitro, will now be used in specific biological tests for each strain of $R$. solani to study their antagonistic capacities in vivo.

Additional key words : Antagonism, mycoparasitism, competition, volatile coumpounds, non-volatile compounds.

\section{INTRODUCTION}

La lutte biologique contre Rhizoctonia solani Kühn, fait largement appel à l'utilisation de l'antagoniste Trichoderma spp. L'efficacité de la lutte dépend du choix de souches antagonistes performantes à partir de critères impliquant une bonne connaissance des particularités biologiques du matériel fongique utilisé. Or le genre Trichoderma et l'espèce $R$. solani ne constituent pas des entités biologiques très fixées et sont donc sujets à de grandes variations au sein de leurs populations.
Pour Trichoderma, dont la systématique est assez aléatoire, les modes d'action antagoniste, bien étudiés par DENNIS \& WEBSTER $(1971, a, b, c)$ peuvent servir à caractériser les souches. Dans le cas de $R$. solani, cette caractérisation est plus précise : les souches de $R$. solani peuvent être distinguées d'autres souches à aspect morphologique identique (PARMETER et al., 1969) et peuvent être classées en groupes biologiquement différents au moyen de l'anastomose de leurs hyphes avec des souches de référence (SCHULTZ, 1937 ; RICHTER \& SCHNEIDER, 1953 ; PARMETER, et al., 1969 ; OGOSHI, 1972, 1976) : 5 groupes d'anasto- 
moses (GA 1 à GA 5) ont ainsi été définis et ont été récemment confirmés par la sérologie (ADAMS \& BUTLER, 1979) et par le spectre des protéines solubles (REYNOLDS et al., 1983) ; ces groupes d'anastomose recouvrent des caractéristiques différentes tant au point de vue spécificité parasitaire que particularités génétiques (ANDERSON, 1982).

Nous avons défini (CAMPOROTA, 1982b) un programme de sélection de souches de Trichoderma spp. pour lutter contre $R$. solani :

- études in vitro et in vivo de l'aptitude antagoniste de souches de Trichoderma et essai de corrélation entre les 2 types de mesures afin de définir des critères rapides de sélection ;

- détermination de la capacité des souches retenues, au terme de ce $1^{\text {er }}$ tri, à coloniser des sols naturels (CAMPOROTA, 1983) ;

- introduction des souches les plus performantes dans des sols infestés par $R$. solani et appréciation du pouvoir protecteur sur la plante (CAMPOROTA, 1982a).

Afin de réaliser la $1^{\text {re }}$ partie de ce programme, nous avons choisi de confronter in vitro 3 souches de $R$. solani bien caractérisées avec 28 souches de Trichoderma spp. pour lesquelles nous avons mesuré individuellement les 3 modes d'action définis par DENNIS \& WEBSTER (1971) : mycoparasitisme, émission de substances agissant sur la croissance, soit volatiles, soit non volatiles. Le but de ce travail est de savoir, d'une part, si les souches de $R$. solani ont une réaction différente face à l'antagonisme global de Trichoderma et, d'autre part, quelle est l'incidence des 3 modes d'action dans le phénomène antagoniste.

\section{MATÉRIEL ET MÉTHODES}

\section{A. Choix du matériel fongique}

\section{Rhizoctonia solani}

Deux raisons ont guidé le choix des 3 souches utilisées dans cette étude :

- elles appartiennent à des groupes d'anastomose (GA) différents : GA $1=\mathrm{Rh} 120$, isolée sur œillet, GA $2=\mathrm{Rh} 15$, isolée sur radis et GA $3=\mathrm{Rh} 328$, isolée sur pomme de terre.

- elles ont un pouvoir pathogène différent sur 3 hôtes végétaux.

Les différences de pouvoir pathogène ont été mises en évidence au moyen de tests biologiques mis au point dans notre laboratoire et utilisant des plantules sensibles (CAMPOROTA, 1980, 1982a; AOUCHICHE, 1982). Les espèces végétales employées présentent, dans chacun des tests, une spécificité relative pour les souches de $R$. solani appartenant à un groupe d'anastomose : les souches classées dans GA 1 sont agressives sur Vigna radiata, celle de GA 2 sur Raphanus sativus tandis que celles de GA 3 attaquent presque uniquement Solanum tuberosum. Le tableau 1 indique les degrés d'attaque obtenus sur ces 3 espèces végétales au moyen des 3 souches de $R$. solani. Il faut noter que ces tests biologiques serviront dans l'étape sui-
TABLEAU 1

Intensité des attaques produites par les 3 souches de R. solani sur 3 hôtes végétaux.

Disease intensities produced by the $3 \mathrm{R}$. solani strains on 3 hosts.

\begin{tabular}{lccc}
\hline \hline & $\begin{array}{c}\text { Vigna } \\
\text { radiata }\end{array}$ & $\begin{array}{c}\text { Raphanus } \\
\text { sativus }\end{array}$ & $\begin{array}{c}\text { Solanum } \\
\text { tuberosum }\end{array}$ \\
\hline Rh 120 & ++++ & +++ & ++ \\
Rh 15 & + & +++ & + \\
Rh 328 & - & - & ++++ \\
\hline
\end{tabular}

++++ : très fortes (very strong); +++ : fortes (strong) ; ++ : faibles (weak) ; + : très faibles (very weak) ; - nulles (no disease)

vante pour juger in vivo du pouvoir antagoniste des souches de Trichoderma spp.

\section{Trichoderma spp.}

Nous avons rassemblé des souches de Trichoderma présentant la plus grande diversité possible; le tableau 2 fournit les numéros de collection, l'espèce, l'origine et la date d'isolement.

Cet ensemble de souches recouvre plusieurs espèces : $T$. harzianum Rifai, réputé pour son efficacité est plus largement représenté (14 souches), T. hamatum Rifai ( 5 souches) et $T$. viride Pers. (3 souches).

Les souches proviennent d'isolements effectués soit sur des champignons pathogènes attaqués, soit directement à partir du sol ; la plupart proviennent des différentes régions de France. Il s'y ajoute une souche d'Israël et 4 souches du Brésil.

Certaines des souches de l'I.N.R.A., Montpellier (DAVET) sont utilisées pour la lutte contre le Sclerotinia de la laitue, celles de l'I.N.R.A., Bordeaux (DUBOS) contre Botrytis cinerea sur la vigne.

Un certain nombre de clones d'une même souche de T. harzianum utilisée par GROSCLAUDE (I.N.R.A., Avignon) pour lutter contre le plomb des arbres fruitiers ont été inclus dans l'expérimentation (souches $\mathrm{T} 23$ à $\mathrm{T} 26, \mathrm{~T} 33$ à $\mathrm{T} 40$ ). Leurs réactions serviront à juger de la stabilité des caractères biologiques de cette souche.

\section{B. Mesure des phénomènes d'antagonisme}

On utilise pour les 2 champignons, des cultures sur milieu malt gélosé (Cristomalt à $1 \mathrm{p} .100$ ), placées en étuve obscure à $23{ }^{\circ} \mathrm{C}$ pendant $8 \mathrm{j}$.

\section{Antagonisme global en confrontation directe}

Dans des boîtes de Petri de $90 \mathrm{~mm}$ de diamètre, contenant $15 \mathrm{ml}$ de milieu malt, on place, à $40 \mathrm{~mm}$ l'un de l'autre, 2 explants de $6 \mathrm{~mm}$ de diamètre provenant des cultures des 2 champignons. Toutes les combinaisons entre les 3 souches de $R$. solani et les 28 souches de l'antagoniste sont réalisées et répétées 2 fois. L'ensemble des boîtes est déposé en étuve obscure à $23{ }^{\circ} \mathrm{C}$ pendant $72 \mathrm{~h}$ au terme desquelles on effectue la notation.

Des observations préliminaires ont montré que, dans l'ensemble des combinaisons, les colonies de Tri- 
TABLEAU 2

Souches de Trichoderma utilisées pour l'expérimentation.

Trichoderma strains used in the experiment.

\begin{tabular}{|c|c|c|c|}
\hline Souches & Espèce & Isolement & Date et origine \\
\hline T 3 & harzianum Pers. & Sclérotes Sclerotinia & 10.79 - INRA Montpellier - DAVET \\
\hline $\mathrm{T} 4$ & harzianum Pers. & Sclérotes Sclerotinia & 10.79 - Israël - HENIS \\
\hline T 18 & viride Rifai & Sol & 3.80 - Muséum Hist. Nat. - Paris \\
\hline Т 19 & hamatum Pers. & Sclérotes Sclerotinia & 3.80 - INRA Montpellier - DAVET \\
\hline Т 21 & koningii Oud. & Sol & 3.80 - INRA Montpellier - DAVET \\
\hline $\begin{array}{l}\text { T } 23 \\
\text { T } 24 \\
\text { T } 25 \\
\text { T } 26\end{array}$ & $\begin{array}{l}\text { harzianum Pers. } \\
\text { harzianum Pers. } \\
\text { harzianum Pers. } \\
\text { harzianum Pers. }\end{array}$ & $\left\{\begin{array}{l}\text { Clones de } \\
\text { la souche } \\
\text { GrosCLAUDE } \\
\left(\mathrm{C}_{2}\right)\end{array}\right.$ & $\{3.80-$ INRA Bordeaux - DUBos \\
\hline Т 29 & hamatum ? Pers. & Sol & \\
\hline $\begin{array}{l}\text { T } 30 \\
\text { T } 31 \\
\text { T } 32\end{array}$ & $\begin{array}{l}\text { hamatum? Pers. } \\
\text { hamatum? Pers. } \\
\text { hamatum? Pers. }\end{array}$ & $\begin{array}{l}\text { Sol } \\
\text { Sol } \\
\text { Sol }\end{array}$ & $\left\{\begin{array}{c}3.81-\text { Brésil Mission Bочнот } \\
1980\end{array}\right.$ \\
\hline $\begin{array}{ll}\text { T } & 33 \\
\text { T } 34 \\
\text { T } 35 \\
\text { T } 36 \\
\text { T } 37 \\
\text { T } 38 \\
\text { T } 39 \\
\text { T } 40\end{array}$ & $\left.\begin{array}{l}\text { harzianum } \text { Pers. } \\
\text { harzianum } \text { Pers. } \\
\text { harzianum } \text { Pers. } \\
\text { harzianum } \text { Pers. } \\
\text { harzianum Pers. } \\
\text { harzianum } \text { Pers. } \\
\text { harzianum Pers. } \\
\text { harzianum Pers. }\end{array}\right\}$ & $\begin{array}{l}\text { Clones de } \\
\text { la souche } \\
\text { GrosCLAUDE } \\
\left(C_{2}\right)\end{array}$ & $\left\{\begin{array}{r}3.81 \text { Contrat d'étude } \\
\text { Société ORSAN }\end{array}\right.$ \\
\hline $\begin{array}{l}\text { T } 41 \\
\text { T } 42\end{array}$ & $\begin{array}{l}\text { viride } \text { Rifai } \\
\text { viride } \text { Rifai }\end{array}$ & $\{$ Clones souche T 18 & $\begin{array}{l}9.81 \text { Conservation azote liquide } 6 \text { mois } \\
5.82 \text { Conservation azote liquide } 12 \text { mois }\end{array}$ \\
\hline $\mathrm{T} 43$ & Indéterminée & Sclérotes Sclerotinia & 2.80 INRA - Dijon \\
\hline T 44 & Indéterminée & Sclérotes Sclerotinia & 2.80 INRA - Dijon \\
\hline T 45 & Indéterminée & Sol & 3.81 INRA - Angers \\
\hline T 46 & Indéterminée & Colonie $R$. solani & 7.82 INRA - Dijon \\
\hline T 47 & Indéterminée & Colonie $R$. solani & 7.82 INRA - Dijon \\
\hline
\end{tabular}

choderma envahissaient celles du pathogène avec une vitesse variable suivant les souches. Nous avons donc estimé que la résultante de la compétition entre les 2 champignons était révélée par la valeur, en pourcentage, de la colonisation par Trichoderma de l'espace séparant les 2 explants.

La mesure de cette colonisation (C) pour chaque souche de Trichoderma est obtenue en faisant le rapport suivant :

$$
\mathrm{C}=\frac{\mathrm{DT} \times 100}{\mathrm{DE}}
$$

où DT est la distance parcourue, sur l'axe reliant les 2 explants, par le front de la colonie de Trichoderma au bout de $72 \mathrm{~h}$ et $\mathrm{DE}$, la distance séparant les 2 explants.

L'expérimentation est répétée 2 fois dans le temps.

\section{Modes d'actions de Trichoderma}

\section{a) Mycoparasitisme}

Dans les boîtes de Petri où sont réalisées les confrontations directes, on observe au microscope $(\mathrm{G} \times 40)$ la zone d'interpénétration des 2 colonies afin d'estimer, pour chaque combinaison, l'intensité du phénomène d'enroulement des hyphes de Trichoderma sur ceux de $R$. solani. On attribue à chacune d'entre elles une note de mycoparasitisme suivant une échelle comprenant 5 valeurs : 0 (pas d'enroulement), $25,50,75,100$ (enroulements intenses).

\section{b) Emission de substances volatiles}

Afin de limiter les pertes en gaz, nous avons fait réaliser une série d'appareils permettant de placer en vis-à-vis 2 fonds de boîtes de Petri (diamètre $90 \mathrm{~mm}$ ), séparées par une cloison percée d'un orifice pouvant être fermé par une trappe coulissante. Les appareils ont été usinés dans du duralumin de façon à être stérilisés avant chaque utilisation.

Dans une boîte de Petri, on dépose 2,5 g de paille de blé hachée à $5 \mathrm{~mm}$ et préalablement désinfectée par 2 passages à $120^{\circ} \mathrm{C}$ pendant $30 \mathrm{mn}$ (OLIVIER \& GERMAIN, 1983). Dans la paille humidifiée par $10 \mathrm{ml}$ d'eau stérile, sont enfouis 9 explants de $6 \mathrm{~mm}$ de diamètre provenant d'une culture de Trichoderma. Le fond de cette boîte est placé dans le logement inférieur d'un appareil décrit plus haut ; la trappe est fermée hermétiquement et l'ensemble, enfermé dans une boîte de Petri de diamètre $160 \mathrm{~mm}$, est soumis pendant $8 \mathrm{j}$ à une température de $23{ }^{\circ} \mathrm{C}$.

Ce temps écoulé, on dépose au centre de boîtes de Petri contenant $15 \mathrm{ml}$ de milieu malt gélosé, un explant de $6 \mathrm{~mm}$ de diamètre provenant de la culture d'une souche de $R$. solani. Cinq de ces boîtes servent de témoin et le fond des autres est placé à la partie supérieure des appareils, la trappe est ouverte et on expose ainsi la souche de $R$. solani à l'influence des 
substances émises par les 28 souches de l'antagoniste pendant $24 \mathrm{~h}$ à l'obscurité et à $23^{\circ} \mathrm{C}$, l'explant de $R$. solani se trouvant à environ $20 \mathrm{~mm}$ au-dessus de la culture de Trichoderma.

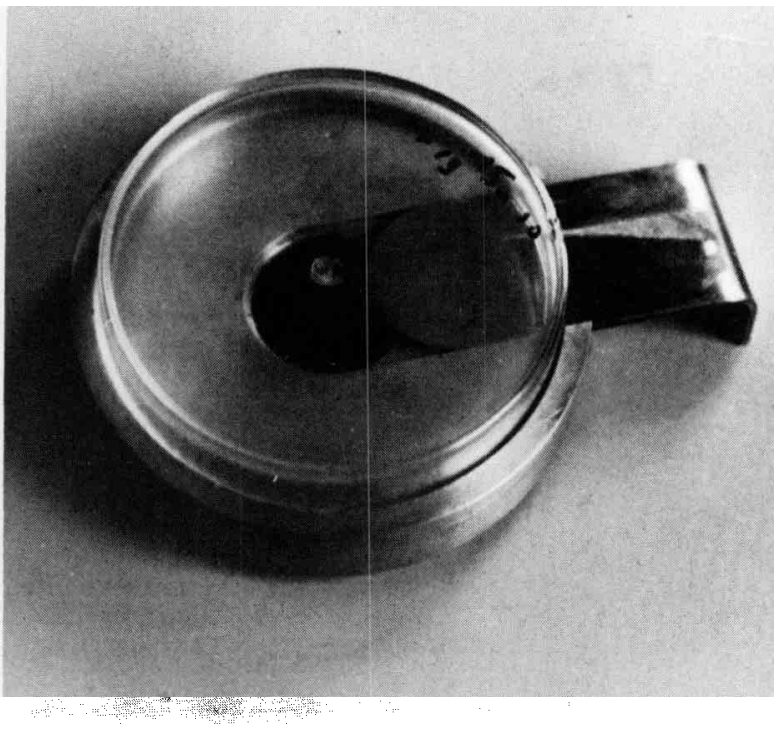

Figure 1

Appareil utilisé pour tester l'influence des substances volatiles émises par Trichoderma sp. sur la croissance de R. solani.

Apparatus used to test the effect of volatile substances emitted by Trichoderma sp. on the growth of $\mathrm{R}$. solani.

On mesure alors le diamètre des colonies de $R$. solani: la vitesse de croissance est calculée en $\mathrm{mm} / \mathrm{h}$ et, par rapport à la croissance des témoins, on obtient, pour chaque combinaison, un pourcentage d'inhibition ou de stimulation de croissance de la souche du pathogène. Cette expérimentation, réalisée pour chaque souche de $R$. solani, est répétée 2 fois dans le temps.

c) Diffusion de substances antagonistes non volatiles dans le milieu de culture

Pour chacune des souches de Trichoderma, on prélève dans des boîtes de culture, des rondelles de $6 \mathrm{~mm}$ de diamètre. Ces rondelles sont déposées au centre de boîtes de Petri contenant $15 \mathrm{ml}$ de milieu malt recouvert, $24 \mathrm{~h}$ auparavant, par une feuille de cellophane stérile. L'ensemble des boîtes demeure $48 \mathrm{~h}$ à l'obscurité en étuve réglée à $23^{\circ} \mathrm{C}$.

$\mathrm{Au}$ bout de ce temps, on soumet les 3 souches de $R$. solani à l'influence des substances diffusées dans le milieu par chaque souche de l'antagoniste : la feuille de cellophane portant l'explant de Trichoderma est retirée et on dépose au centre de la boîte, à raison de 2 répétitions par souche du pathogène, un explant de $6 \mathrm{~mm}$ de diamètre. Dans le même temps, 5 boîtes de milieu malt inoculées par chacune des souches de $R$. solani servent de témoin de croissance.

L'ensemble des combinaisons est réalisé et les boîtes sont ensuite soumises pendant $24 \mathrm{~h}$ aux mêmes conditions d'environnement que précédemment. La mesure du diamètre des colonies permet de calculer, en pourcentage, l'inhibition de croissance des colonies de $R$. solani. L'expérimentation est répétée 2 fois dans le temps.

\section{RÉSULTATS}

\section{A. Antagonisme global}

Les résultats de la confrontation directe entre les souches des 2 champignons sont présentés sur la figure 2 (regroupant 3 graphiques: Rh 120-Rh 328$\mathrm{Rh}$ 15) dans laquelle l'axe des ordonnées porte l'intensité du phénomène de colonisation $(C$, exprimée en pourcentage) et l'axe des abscisses, l'ensemble des souches de Trichoderma repérées par leur numéro de collection.

Pour chaque souche de $R$. solani, les valeurs de C sont la résultante de l'interaction entre l'aptitude de Trichoderma à envahir $R$. solani et la capacité de ce dernier à s'y opposer. L'ensemble des résultats obtenus montre que la souche $\mathrm{Rh} 15$ semble offrir un milieu plus favorable à l'envahissement que $\mathrm{Rh} 120$ et $\mathrm{Rh}$ 328. Le calcul de la moyenne des valeurs de $\mathrm{C}$ pour chaque souche de $R$. solani confirme cette observation : la moyenne de $C$ pour $\mathrm{Rh} 15$ est significativement différente (au seuil de 5 p. 100) de celles de $\mathrm{Rh} 120$ et $\mathrm{Rh} 328$ qui ne se distinguent pas l'une de l'autre (tabl. 3).

La réaction des différents clones de la souche T. harzianum de GROSCLAUDE, T 23 à T 26 et T 33 à T 40 (cf. II, A 1), diffère en fonction de la souche de $R$. solani à laquelle ils sont opposés, par contre, elle est sensiblement analogue pour une même souche du parasite.

Mais la mesure de $C$ ne rend pas compte d'un phénomène général que nous avons observé tout au long de l'expérimentation : la confrontation de Trichoderma avec $\mathrm{Rh} 120$ et $\mathrm{Rh} 328$ aboutit à la lyse de la colonie du parasite en 5 à $6 \mathrm{j}$ en moyenne tandis que le même résultat n'est obtenu qu'en 8 à $9 \mathrm{j}$ dans le cas de $\mathrm{Rh} 15$.

\section{TABLEAU 3}

Moyenne des valeurs de colonisation (C) mesurées pour chaque souche de R. solani.

Mean of the colonization values (C) measured for each R. solani strain.

\begin{tabular}{lccc}
\hline & Rh 120 & Rh 328 & Rh 15 \\
\hline $\bar{X} \mathrm{C}$ & 66 & 72,8 & 85,9 \\
\hline $\begin{array}{l}\text { Intervalle de confiance } \\
\text { à } 5 \text { p. } 100\end{array}$ & 6,13 & 5,68 & 5,40 \\
\hline
\end{tabular}

\section{B. Modes d'action}

Les mesures effectuées pour les 3 modes d'action sont présentées sur les figures 3 (Mycoparasitisme, M), 4 (Substances volatiles, SV) et 5 (Substances non volatiles, SNV). Comme précédemment, chaque figure regroupe les résultats correspondant aux 3 souches de $R$. solani : l'axe des ordonnées porte l'intensité du phénomène antagoniste et l'axe des abscisses les sou- 
\% colonisation(C)
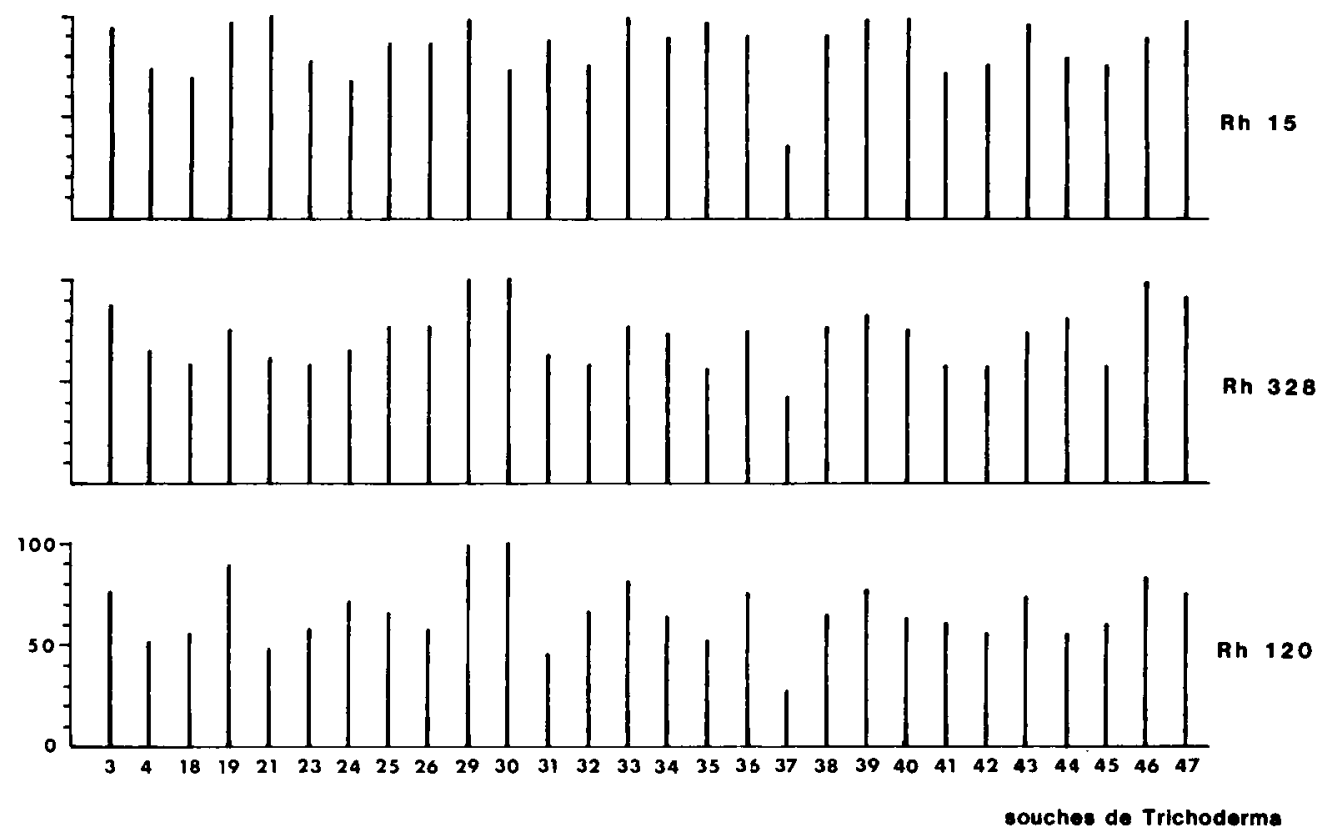

Figure 2

Intensités de colonisation (C), en pourcentage des 3 souches de $\mathrm{R}$. solani (Rh $120 ; R h 328 ; R h$ 15) par chaque souche de Trichoderma.

Intensity of colonization $(C)$, expressed as \% for the $3 \mathrm{R}$. solani strains

(Rh $120 ; R h 328 ; R h$ 15) with each strain of Trichoderma.

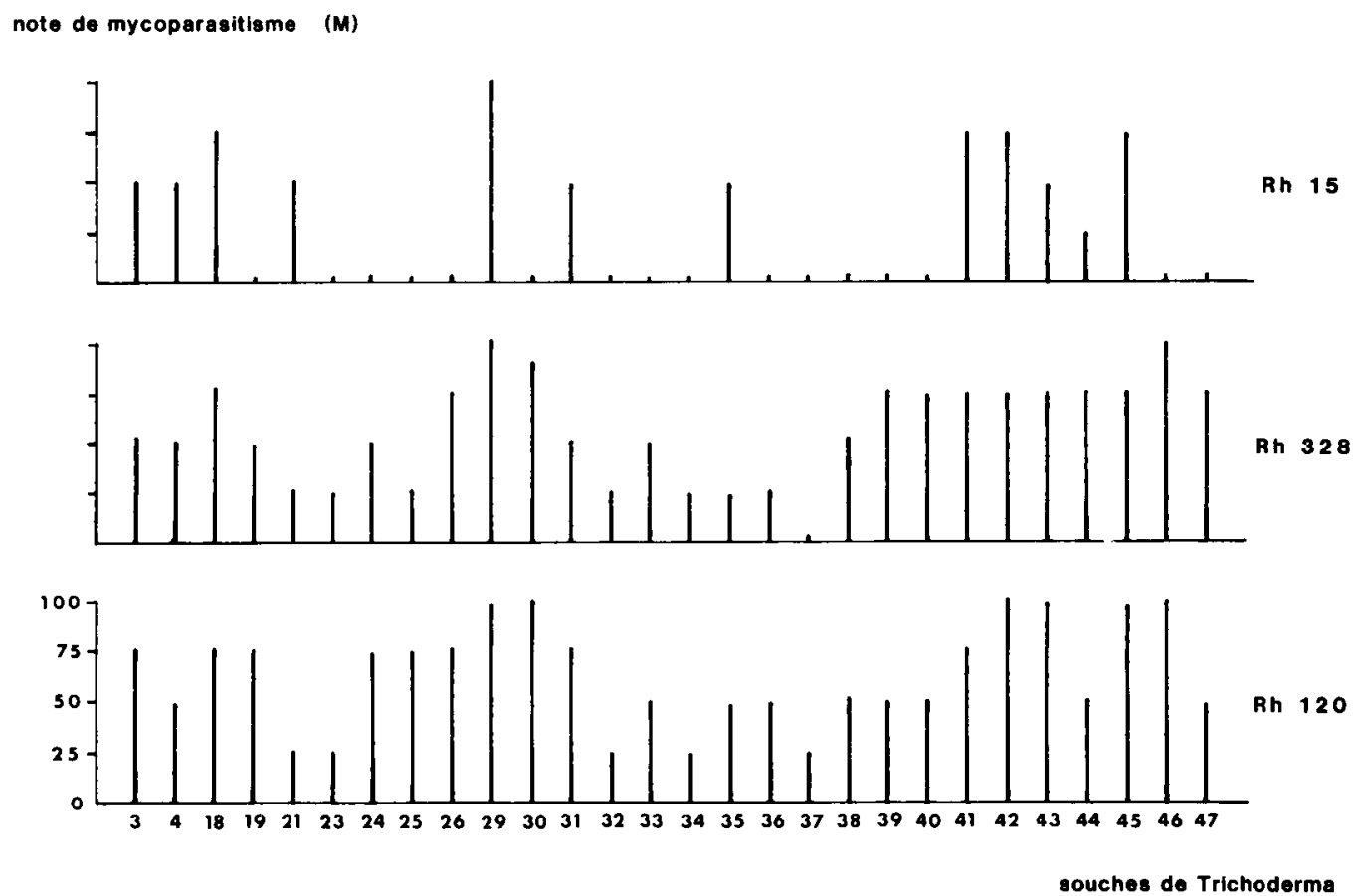

Figure 3

Note de réaction au mycoparasitisme (M) attribuée à chaque souche de $\mathrm{R}$. solani (Rh $120 ; R h 328 ; R h$ 15) confrontée aux 28 souches de Trichoderma.

Mycoparasitism score (M) given to each R. solani strain (Rh 120;

$R h 328 ; R h 15)$ confronted with the 28 Trichoderma strains. 


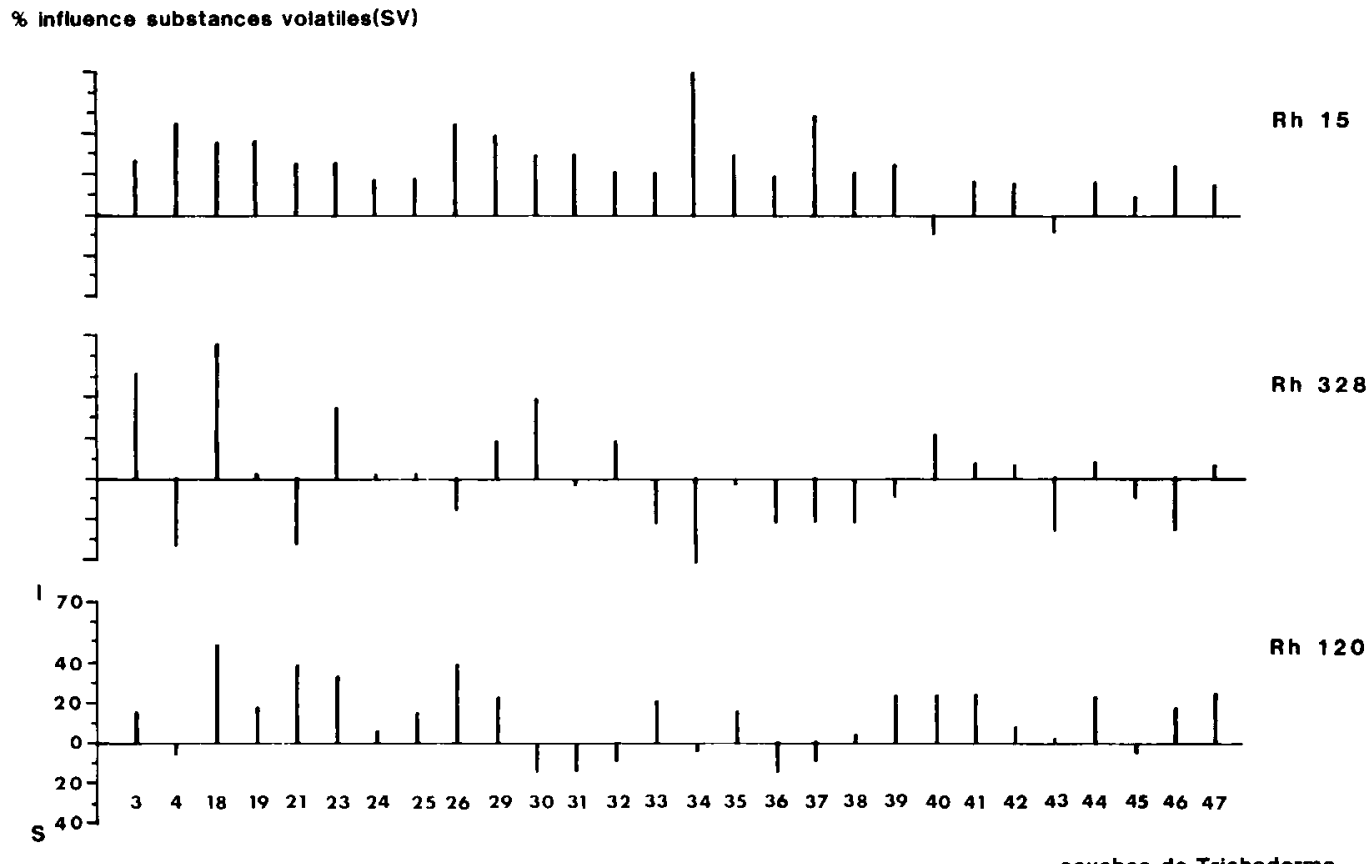

Figure 4

Pourcentages d'inhibition (I) ou de stimulation (S) de la croissance des 3 souches de $\mathrm{R}$. solani ( $R$ h $120 ; R h 328 ; R h$ 15) sous l'effet de substances volatiles (SV) émises par chaque souche de Trichoderma.

Percent of growth inhibition (I) or stimulation (S) of the 3 R. solani strains ( $R h$ 120; $R h$ 328; $R h$ 15) by volatile compounds (SV) produced by each strain of Trichoderma.

\% influence substances non volatiles (SNV)
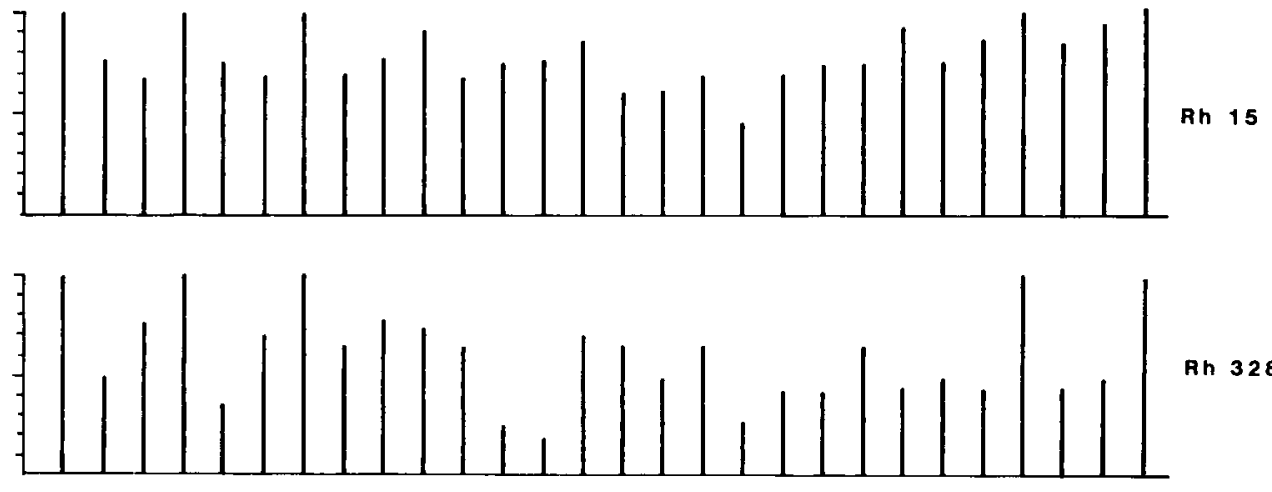

Rh 328

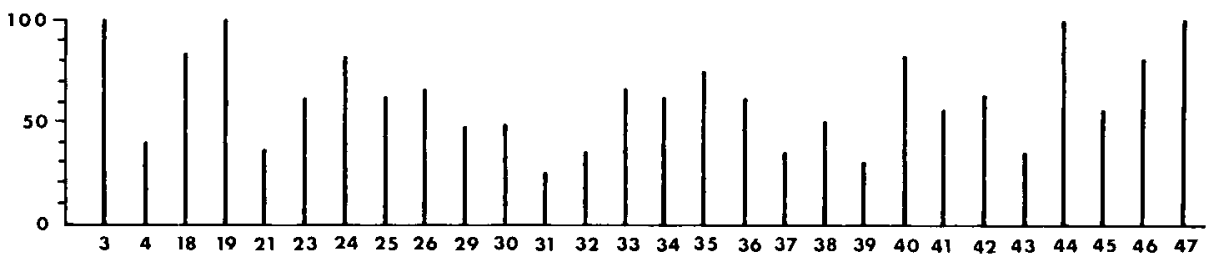

Rh 120

souches de Trichoderma

Figure 5

Pourcentages d'inhibition de la croissance des 3 souches de $\mathrm{R}$. solani ( $R$ h $120 ; R h 328 ; R h$ 15) sous l'effet de substances non volatiles (SNV) diffusées dans le milieu par chaque souche de Trichoderma.

Percent of growth inhibition of the 3 R. solani strains (Rh 120; $R h 328$; $R h$ 15) by non volatile compounds (SNV) released in the medium by each strain of Trichoderma. 
ches de Trichoderma repérées par leur numéro de collection.

Les différences de réaction des 3 souches de $R$. solani sont relativement nettes : Rh 15 se distingue des 2 autres par une sensibilité moindre au mycoparasitisme ; elle est, par contre, plus touchée par l'action des substances volatiles. Il est à remarquer qu'à l'inverse des 2 autres souches, ce dernier mode d'action ne provoque pas chez Rh 15 de phénomène de stimulation de croissance. La distinction entre les réactions de $\mathrm{Rh} 120$ et $\mathrm{Rh} 328$ est beaucoup moins perceptible, si ce n'est pour l'influence des substances volatiles.

Par ailleurs, la conservation à l'azote liquide (cf. tabl. 2) a entraîné une baisse importante de l'émission des substances volatiles par les 2 souches T 41 et T 42 par rapport à la souche mère $\mathrm{T} 18$.

L'observation microscopique de la zone d'interpénétration des 2 colonies pour apprécier le mycoparasitisme (cf. Matériel et Méthodes, $\S$ B 2a) confirme encore les divergences de réaction des 3 souches de $R$. solani : le phénomène de mycoparasitisme (vacuolisation du cytoplasme du parasite, enroulement des hyphes de Trichoderma, cassure des hyphes de $R$. solani au niveau des cloisons et enfin lyse complète des hyphes) se constate surtout pour les souches $\mathrm{Rh} 120$ et Rh 328. Dans le cas de Rh 15, le mycoparasitisme est peu important mais on constate surtout un mélange des hyphes des 2 champignons, sans contact et apparemment sans réaction, pendant 6 à $7 \mathrm{j}$ en moyenne ; puis, brusquement, survient un flétrissement des hyphes du pathogène qui disparaissent ensuite complètement. Considérant que l'élimination de $R$. solani survenait dans les 2 cas, bien que le mycoparasitisme soit plus rapidement efficace, nous n'avons pas fait de notation distincte des 2 phénomènes.

\section{Incidence des modes d'action dans I'antagonisme global}

Nous avons estimé que les valeurs de $C$ (intensité de colonisation) rendaient compte du résultat de l'antagonisme entre $R$. solani et Trichoderma. Pour savoir si les différences de sensibilité à l'envahissement, observées pour chaque souche de $R$. solani, pouvaient être expliquées en fonction des 3 modes d'action, des corrélations ont été recherchées entre $C$, variable à expliquer, et $\mathrm{M}, \mathrm{SNV}, \mathrm{SV}$, variables explicatives, au moyen d'un programme progressif de calcul de régressions multiples. Les valeurs des 4 paramètres mesurés pour chaque souche de $R$. solani ont été introduites dans un ordinateur (Mini 6, Unité informatique, I.N.R.A., Dijon) qui, par confrontations, a fourni une analyse en 3 niveaux rendant compte d'une force de liaison décroissante du niveau 1 au niveau 3 (BACHACOU et al., 1981), (tabl. 4).

On constate une différence entre les 3 souches de $R$. solani : les valeurs de $C$ sont positivement et significativement liées aux valeurs des paramètres $\mathbf{M}$ et SNV pour la souche $\mathrm{Rh} 328$, à celles de $M$ pour $\mathrm{Rh} 120$ et à celles de SNV pour le $\mathrm{Rh} 15$. Dans les 3 cas, la liaison négative de $C$ avec les valeurs du paramètre $\mathrm{SV}$ semble indiquer que ce mode d'action n'a pas d'incidence importante dans le phénomène de colonisation.
TABLEAU 4

Corrélations entre les valeurs de colonisation (C) et celles des 3 modes d'action pour chaque souche de $\mathbf{R}$. solani : $M=$ mycoparasitisme $; S V=$ substances volatiles ; $S N V=$ substances non volatiles.

Correlations obtained between colonization values $(C)$ and the values of the 3 mechanisms of antagonism for each strain of R. solani : $M=$ mycoparasitism $; S V=$ volatile compounds ; $S N V=$ non-volatile compounds.

\begin{tabular}{|c|c|c|c|c|}
\hline Souche & $\begin{array}{l}\text { Niveau } \\
\text { de liaison }\end{array}$ & $\begin{array}{l}\text { Variable } \\
\text { introduite }\end{array}$ & $\begin{array}{l}\text { Coefficient } \\
\text { de corrélation }\end{array}$ & $\begin{array}{l}\text { Test } \mathrm{t} \text { de } \\
\text { Student }\end{array}$ \\
\hline \multirow{6}{*}{ Rh 328} & 1 & M & 0,338 & $3,486^{* *}$ \\
\hline & \multirow[t]{2}{*}{2} & M & 0,305 & $3,386^{* *}$ \\
\hline & & & & \\
\hline & \multirow[t]{3}{*}{3} & $\mathbf{M}$ & 0,317 & $3,446^{* *}$ \\
\hline & & SNV & 0,269 & $2,523^{*}$ \\
\hline & & SV & $-0,841$ & $-0,801$ \\
\hline \multirow{6}{*}{ Rh 120} & 1 & $\mathrm{M}$ & 0,321 & $2,810^{* *}$ \\
\hline & \multirow[t]{2}{*}{2} & $\mathbf{M}$ & 0,309 & $2,745^{*}$ \\
\hline & & SNV & 0,178 & 1,451 \\
\hline & \multirow[t]{3}{*}{3} & M & 0,300 & $2,633^{*}$ \\
\hline & & SNV & 0,220 & 1,636 \\
\hline & & SV & $-0,152$ & $-0,794$ \\
\hline \multirow{6}{*}{ Rh 15} & 1 & SNV & 0,470 & $2,286^{*}$ \\
\hline & \multirow[t]{2}{*}{2} & SNV & 0,499 & 2,329 \\
\hline & & $\mathbf{M}$ & $-0,515$ & $-0,576$ \\
\hline & \multirow[t]{3}{*}{3} & SNV & 0,448 & 1,878 \\
\hline & & M & $-0,533$ & $-0,587$ \\
\hline & & SV & $-0,100$ & $-0,504$ \\
\hline
\end{tabular}

Le test « $t$ » de STUDENT indique la signification des coefficients de corrélation : ** hautement significatif ; ${ }^{*}$ significatif.

Student's « $t$ » test for significance level : ${ }^{* *}$ highly significant ; * significant.

\section{DISCUSSION}

Les résultats présentés montrent qu'il existe non seulement des différences de sensibilité des 3 souches de $R$. solani à l'antagonisme par Trichoderma mais également que chacune des souches de l'antagoniste possède une aptitude particulière pour éliminer l'agent pathogène. Les souches de $R$. solani les plus sensibles au mycoparasitisme sont celles qui sont le plus rapidement détruites : c'est le cas de Rh 120 et surtout de $\mathrm{Rh} 328$ où la liaison de $\mathrm{C}$ avec $\mathrm{M}$ et $\mathrm{SNV}$ semble impliquer une action synergique entre les 2 modes d'action. La souche $\mathrm{Rh} 15$, pour laquelle $\mathrm{C}$ est seulement lié avec SNV, est détruite au bout d'un temps plus long ; cela signifierait que l'efficacité de ce mode d'action est inférieure à celle du mycoparasitisme.

Dans une récente communication, CHET (1984) a fait état d'études sur les modes d'action de Trichoderma utilisé pour la lutte biologique contre $R$. solani dans le cas de la culture du cotonnier et du fraisier : les résultats ont mis en évidence l'importance du phénomène de mycoparasitisme dans l'efficacité de Trichoderma. Nous pensons, nous aussi, que les souches antagonistes les plus efficaces sont celles qui, du fait de leur capacité à entrer en contact étroit avec les 
hyphes de $R$. solani, favorisent l'action des enzymes ( $\beta 1$-3-glucanase, chitinase) conduisant à la lyse du mycélium du parasite.

Ce même auteur a montré que la «reconnaissance " des hyphes du parasite par ceux de l'antagoniste était le résultat d'une liaison entre des lectines présentes sur les hyphes de $R$. solani et de sucres (D ou L glucose et D ou L galactose) portés par les hyphes de Trichoderma (CHET \& ELAD, 1983). De ce point de vue, un fait très intéressant se dégage de nos résultats : quand on considère les valeurs du paramètre M (cf. fig. 3) on voit que certaines souches de Trichoderma parasitent indifféremment les 3 souches de $R$. solani (souches T 29, T 3, T 4, T 18, T 41, T 42, $\mathrm{T}$ 43), tandis que d'autres n'attaquent que $\mathrm{Rh} 120$ et Rh 328 (T 19, T 24, T 30, T 46, T 47). Nous pensons que ces souches, associées à celles de $R$. solani, constituent un matériel de choix pour des études fines de confirmation et de compréhension du phénomène de liaison lectines-sucres. A terme, cela pourrait conduire à améliorer les souches de Trichoderma par mutations génétiques et à posséder ainsi un matériel plus efficace pouvant éventuellement être utilisé contre d'autres champignons parasites du sol.

Les résultats que nous avons rapportés sont la $1^{\text {re }}$ étape du programme de sélection entrepris : nous possédons une meilleure connaissance des particularités biologiques des souches de $R$. solani et Trichoderma. $\mathrm{La} 2^{\mathrm{e}}$ étape consiste maintenant, en utilisant les mêmes souches, à apprécier in vivo l'efficacité des souches antagonistes au moyen de la mesure d'un niveau de maladie provoqué par les 3 souches de $R$. solani dans les biotests spécifiques. Les résultats in vitro et in vivo seront ensuite confrontés et si nous aboutissons à la conclusion que des phénomènes d'antagonisme mesurés in vitro correspondent à une réalité au niveau de la maladie sur la plante, nous serons en mesure de progresser beaucoup plus rapidement dans la sélection de souches de Trichoderma performantes pour une lutte intégrée contre $R$. solani dont l'importance en culture maraîchère ne cesse de croître ces dernières années.

Reçu le 19 novembre 1984 Accepté le 11 mars 1985.

\section{REMERCIEMENTS}

L'auteur tient à remercier Mme R. CAMPOROTA pour l'exécution des figures de ce texte, M. A. CARTERon (Amélioration des Plantes, Dijon) pour l'exploitation statistique des résultats sur ordinateur, MM. S. Almanza et R. Renaud (Génie Microbiologique, Dijon) pour la réalisation des appareils de mesure pour les substances volatiles, enfin le Professeur E. E. ButLer (Université de Californie) pour la fourniture des souches de référence de $R$. solani.

\section{RÉFÉRENCES BIBLIOGRAPHIQUES}

Adams G. C., Butler E. E., 1979. Serological relationships among anastomosis groups of Rhizoctonia solani. Phytopathology, 69 (6), 629-633.

Anderson N. A., 1982. The genetics and pathology of Rhizoctonia solani. Annu. Rev. Phytopathol., 20, 329-347.

Aouchiche H., 1982. Recherche d'une méthode de mesure du poten tiel infectieux de sols infestés par Rhizoctonia solani Kühn agent du Rhizoctone brun de la pomme de terre. D.E.A. "Protection des cultures ", E.N.S.A. de Rennes, 43 p.

Bachacou J., Masson J. P., Millier C., 1981. Programme de régression linéaire progressive. In « Manuel de la programmathèque statistique AMANCE ", 209-249. C.N.R.F., I.N.R.A., Champenoux.

Camporota P., 1980. Recherches sur l'écologie des champignons parasites dans le sol. XV. Choix d'une plante piège et caractérisation des souches de Rhizoctonia solani pour la mesure du potentiel infectieux des sols et substrats. Ann. Phytopathol., 12 (1), 31-44.

Camporota P., 1982a. Recherches sur l'écologie des champignons parasites dans le sol. XVII. Mesure du potentiel infectieux de sols et substrats infestés par Rhizoctonia solani Kühn, agent de fontes de semis. Agronomie, 2, (5), 437-442.

Camporota P., 1982b. Lutte biologique contre les maladies des plantes induites par des champignons phytopathogènes telluriques. Critères de choix des microorganismes antagonistes : application à Trichoderma sp. 171-177. In « La sélection des plantes pour la résistance aux maladies ", Bordeaux, mars 1982, Coll. de l'I.N.R.A., 11, 171-179.

Camporota P., 1983. Note. Evolution du comportement saprophytique de souches de Trichoderma spp. dans les sols. Technique d'étude. Agronomie, 3 (6), 607-609.

Chet I., 1984. Application of Trichoderma as a biocontrol agent. Proc. 6th Congr. Un. Phytopathol. mediterr., Cairo, Egypt, 110 111.

Chet I., Elad Y., 1983. Mechanism of mycoparasitism. 35-40. In «Les antagonismes microbiens. Mode d'action et application à la lutte biologique contre les maladies des plantes ". Bordeaux, mai 1983, Coll. de l'I.N.R.A., 18, 35-40.

Dennis C., Webster J., 1971 $a$. Antagonistic properties of species groups of Trichoderma. I. Production of non-volatile antibiotics. Trans. Br. Mycol. Soc., 57 (1), 25-39.

Dennis C., Webster J., 1971b. Antagonistic properties of speciesgroups of Trichoderma. II. Production of volatile antibiotics. Trans. Br. Mycol. Soc., 57 (1), 41-48.

Dennis C., Webster J., 1971c. Antagonistic properties of speciesgroup of Trichoderma. III. Hyphal interaction. Trans. Br. Mycol. Soc., 57 (3), 363-369.

Ogoshi A., 1972. Some characters of hyphal anastomosis groups in Rhizoctonia solani. Ann. Phytopathol. Soc. Jpn., 38, 123-129.

Ogoshi A., 1976. Studies on the grouping of Rhizoctonia solani Kühn with hyphal anatomosis and on the perfect stages of groups. Bull. nat. Inst. agric. Sci. Sec. C. Plant Pathol. Entomol., 30, 1 163.

Olivier J. M., Germain R., 1983. Etude des antibiotiques volatils des Trichoderma. 17-34. In «Les antagonismes microbiens. Modes d'action et application à la lutte biologique contre les maladies des plantes ». Bordeaux, mai 1983, Coll. de l'I.N.R.A., 18, 17-34.

Parmeter J. R. Jr, Sherwood R. T., Platt W. D., 1969. Anastomosis grouping among isolates of Thanatephorus cucumeris. Phytopathology, 59, 1270-1278.

Reynolds M., Weinhold A. R., Morris T. J., 1983. Comparison of anastomosis groups of Rhizoctonia solani by polyacrylamide gel electrophoresis of soluble proteins. Phytopathology, 73 (6), 903 906.

Richter H., Schneider R., 1953. Untersuchungen zur morphologischen und biologischen Differenzierung von Rhizoctonia solani $\mathrm{K}$ Phytopathol. Z., 20, 167-226.

Schultz H., 1937. Vergleichende Untersuchungen zur Okologie, Morphologie, und Systematik des « Vermehrungpilzes ». Arb. Biol. Reichanst, für Land-und Forstwirtsch., Berlin, 22, 1-41. 